\title{
Positive and negative signals in mast cell activation
}

DOI:

10.1016/j.it.2017.01.008

\section{Document Version}

Accepted author manuscript

Link to publication record in Manchester Research Explorer

\section{Citation for published version (APA):}

Bulfone-Paus, S., \& Howlett, S. (2017). Positive and negative signals in mast cell activation. Trends in Immunology, 38(9), 657-667. https://doi.org/10.1016/j.it.2017.01.008

\section{Published in:}

Trends in Immunology

\section{Citing this paper}

Please note that where the full-text provided on Manchester Research Explorer is the Author Accepted Manuscript or Proof version this may differ from the final Published version. If citing, it is advised that you check and use the publisher's definitive version.

\section{General rights}

Copyright and moral rights for the publications made accessible in the Research Explorer are retained by the authors and/or other copyright owners and it is a condition of accessing publications that users recognise and abide by the legal requirements associated with these rights.

\section{Takedown policy}

If you believe that this document breaches copyright please refer to the University of Manchester's Takedown Procedures [http://man.ac.uk/04Y6Bo] or contact uml.scholarlycommunications@manchester.ac.uk providing relevant details, so we can investigate your claim.

\section{OPEN ACCESS}




\title{
Trends in Immunology
}

\section{Invited Review manuscript}

\section{Positive and Negative signals in Mast Cell Activation}

\author{
Silvia Bulfone-Paus ${ }^{1}$, Gunnar Nilsson ${ }^{2}$, Petr Draber ${ }^{3}$, Ulrich Blank ${ }^{4,5,6}$, Francesca Levi-Schaffer ${ }^{7}$ \\ ${ }^{1}$ Division of Musculoskeletal and Dermatological Sciences, Faculty of Biology, Medicine and Health, \\ University of Manchester, Manchester, UK \\ ${ }^{2}$ Immunology and Allergy unit, Department of Medicine, Karolinska Institutet and Karolinska \\ University Hospital, Stockholm; and Department of Medical Sciences, Uppsala University, Uppsala, \\ Sweden \\ ${ }^{3}$ Department of Signal Transduction, Institute of Molecular Genetics, Academy of Sciences of the \\ Czech Republic, Prague, Czech Republic \\ ${ }^{4}$ INSERM U1149, Centre de Recherche sur l'Inflammation, ${ }^{5}$ CNRS ERL8252, ${ }^{6}$ Université Paris Diderot, \\ Sorbonne Paris Cité, Faculté de Médecine, Site Xavier Bichat, Inflamex Laboratory of Excellence, \\ Paris, France \\ ${ }^{7}$ Pharmacology and Experimental Therapeutics Unit, School of Pharmacy, Institute for Drug Research, \\ Faculty of Medicine, Hebrew University of Jerusalem, Jerusalem, Israel
}

\begin{abstract}
Mast cells are powerful immune modulators of the tissue microenvironment. Within seconds of activation, mast cells release a great variety of preformed biologically active products, followed by a wave of mediator synthesis and secretion. Increasing evidence suggests that an intricate network of inhibitory and activating receptors, specific signalling pathways and adaptor proteins governs mast cell responsiveness to stimuli. Here, we discuss the biological and clinical relevance of negative and positive signalling modalities that control mast cell activation, with emphasis on novel FceRI-regulators, IgE-independent pathways (e.g., MRGX2 receptor), tetraspanins and the CD300 family of inhibitory and activating receptors.
\end{abstract}




\section{Fine Tuning Mast Cell Activities}

Mature mast cells are heterogeneous, tissue-resident, long-lived, granulated cells, which increase in number in inflammatory conditions by proliferation, increased recruitment, survival and/or maturation from mast cell progenitors. Particularly abundant at barrier sites, mast cells are among the fastest immune cell responders. They rapidly release preformed mediators, such as vasoactive amines (histamine and serotonin), proteoglycans, proteases, and cytokines from intracellular stores upon crosslinking of FcERI (see glossary) bound immunoglobulin E (IgE) by allergens, infectious agents and toxins. Furthermore, mast cells, activated in an IgE-independent manner, shape their tissue microenvironment, engage in cell-cell interactions and contribute to the defence against, and clearance of various pathogens by secreting a wide array of de novo synthesized pro-inflammatory and antiinflammatory products [1-3].

The molecular regulation of mast cell activation by Fc\&RI-mediated signalling via immunoreceptor tyrosine-based activation motifs (ITAMs) has been investigated in depth in the past. Inhibitory receptors, generally characterized by the presence of immunoreceptor tyrosine-based inhibitory motifs (ITIMs which recruit SH2-domain containing phosphatases, have been identified as cardinal regulators of mast cell activation. Among them are the inhibitory low-affinity IgG receptor FcyRIIB, the CD200R and a number of Ig-like receptors [4]. However, other signals, including non-ITIM containing inhibitory receptors (e.g. catecholamine-binding, $\beta 2$-adrenergic, and ATP or adenosine-binding, purinergic, receptors) as well as anti-inflammatory cytokines (e.g. IL-10 and TGF $\beta$ ) can also antagonize mast cell activation and mediator secretion $[5,6]$, underlining the complex regulation of signalling in these cells.

In this review we focus our attention on novel mechanisms that act at various levels and by diverse means, to adjust mast cell activities in order to tune inflammation up or down as needed to avoid mast cell disorders such as allergy, mastocytosis and autoimmunity. 


\section{FcERI-Mediated Signalling: an Intricacy of Positive Signalling and Inhibitory Players}

Evidence has accumulated in recent years introducing the novel concept that (patho)physiological IgE responses vary between individuals and that a number of parameters fine-tune mast cell responsiveness: polyclonality of the IgE repertoire, number of epitopes recognized, their distance from each other and antigen affinity [7, 8]. Moreover, it has become clear that autoantibodies (anti-lgE or IgE) may impact on pathology development [9-11].

It was established early on that aggregates of receptor bound IgE as small as a dimer were sufficient to induce secretory responses [12]. Although this was questioned by the discovery that monomeric IgE can induce mast cell survival and potentiation of cytokine responses [13, 14] follow-up studies demonstrated that the effects of monomeric IgE are caused by aggregation that could be prevented in the presence of carrier-free hapten [15]. Another study examined secretory responses as a function of antigen concentration and showed that low antigen concentrations favoured chemokine secretion in the absence of visible degranulation, while at high antigen concentrations both responses became apparent ([16] and Figure 1A, left). Weak stimulation favoured the phosphorylation of GRB2-associated protein binding 2 (GAB2) and p38 mitogen-activated protein kinase (MAPK), while linker for activation of T cells (LAT1) and extracellular-signal regulated kinase 2 (ERK2) phosphorylation was favoured by a stronger stimulus. In allergic patients, mast cell degranulation induced by allergens was shown to poorly correlate with the allergen-specific IgE content [17]. These studies have challenged the simplistic view of a unique positive forward response and support the concept that the outcome of receptor triggering can be quite variable. They argue against a kinetic proofreading mechanism controlled by the appropriate lifetime of receptor clusters for a given functional response [18]. Rather they favour the hypothesis of a differential coupling of signalling effectors in response to antigen. Such differential molecular editing was demonstrated in carefully controlled analysis of responses to antigens of different affinities [19]. Triggering of FceRI-bound monoclonal IgE upon interaction with a low affinity antigen (adjusted to a similar total tyrosine phosphorylation response) differed from responses observed with the high affinity antigen, namely favouring chemokine secretion over degranulation and cytokine production (Figure $1 \mathrm{~A}$, right). At the same time the dynamics and mobility of FcERI clusters were markedly reduced with aggregated 
receptors exhibiting a differential spatial distribution. Low affinity antigen binding promoted adapter LAT2 phosphorylation instead of LAT1 and favoured recruitment of the Src family kinase FGR, while recruitment of the kinase LYN remained unaltered. In vivo, during a passive cutaneous anaphylaxis response, this differential responsiveness translated to an altered cell recruitment at the inflammatory site. Besides antigen concentrations and affinity, the distance of epitopes recognized by IgE is also a crucial parameter [20]. This was shown by engineering epitopes onto a protein carrier at different distances. This resulted in different shapes of the resulting IgE immune complexes as examined by negative-stain electron microscopy. The study confirmed that the magnitude of functional responses was greater with antigens of higher valency [21], but also that close proximity also increased the potency of the response.

Importantly, many studies demonstrated that FceRI triggering can also deliver an inhibitory response. Indeed, challenge of mast cells under permissive conditions with increasing amounts of low dose antigen rapidly induces desensitization, a principle exploited in clinics to rapidly desensitize patients, notably to drugs $[22,23]$. Although receptor internalization was initially proposed as a causative mechanism [22], later analysis convincingly showed that desensitization rather affected intrinsic signalling responses, including phosphorylation of STAT6 and p38 MAPK, calcium mobilization as well as late responses such as lipid mediator or chemokine/cytokine release [24]. Recent signalling analyses have now shown that certain events such as LYN and LAT1 phosphorylation are still intact, while the calcium response is completely blunted due to a defect in actin rearrangement known to be required for calcium mobilization [25, 26]. Desensitization is antigen specific, since stimulation with a different antigen still triggers responses in sensitized cells $[24,26]$. However, using a procedure targeting all cell surface receptors with anti-FceRI monoclonal antibodies (mAbs), desensitisation can be achieved for a broad range of antigens [27]. Desensitization under low valency conditions has previously been observed for related IgA and IgG Fc receptors, a phenomenon described as inhibitory ITAM (ITAMi) signalling [28, 29]. However, it still remains to be investigated whether this mechanism is operative in FceRI desensitisation and whether it can be inhibitory on heterologous receptors to deliver powerful general antiinflammatory effects as demonstrated for ITAMi signalling [28, 30, 31]. In addition to the direct regulation of signalling at the level of Fc\&RI, many other surface receptors and 
molecules have been described as modulators of FceRI function in various pathological or physiological applications, some of which are discussed in the following sections.

\section{Promiscuous Receptors Governing Mast Cell Activation: the Tetraspanin Family}

FceRIs are compartmentalized in specialized plasma membrane microdomains with actinrestricted diffusion, which facilitate antigen-induced receptor immobilization [32]. The tetraspanin family of receptors, a large group of ubiquitously and abundantly expressed integral membrane proteins, have been shown to contribute to the architectural organization of such domains. Tetraspanins are four-pass transmembrane proteins that share a tight rod-like structure with 200-300 amino acids, a small extracellular domain and a large extracellular domain of variable length and sequence. The transmembrane helices are involved in interactions of tetraspanins with a broad array of other molecules in the plasma membrane, and formation of tetraspanin-enriched microdomains. Tetraspanins appear to have many roles in various areas of cell biology, including cell motility, exosome formation and functions as well as regulation of immunoreceptor signalling [33-35]. In mast cells four tetraspanins, CD9, CD63, CD81, and CD151 (Figure 1B), have been studied using mAbs, knockout mouse models or knockdown cell lines. The results showed that these tetraspanins could have positive or negative regulatory roles on mast cell signalling, depending on the tetraspanin and signalling pathway studied (Fig. 1B).

The first notion that tetraspanins could regulate signalling pathways in mast cells came from experiments in which anti-CD81 mAb blocked antigen-mediated degranulation in rat basophilic leukaemia cells and passive cutaneous anaphylaxis (PCA), a classical system for studying mast cell activation in vivo. Interestingly, anti-CD81 had no effect on antigeninduced tyrosine phosphorylation of numerous substrates, calcium mobilization and leukotriene C4 production [36]. Another anti-tetraspanin mAb, anti-CD63, also inhibited degranulation without any effect on global tyrosine phosphorylation and calcium response, but impaired the GAB2-phosphatidyl inositol 3 kinase (PI3K) pathway, that is known to be essential for both degranulation and adhesion. The inhibitory effect of anti-CD63 was observed in adherent cells, but not in cells in suspension, suggesting that CD63-integrinfibronectin axis is involved in the inhibition [37]. When mouse bone marrow-derived mast cells (BMMCs) from CD63-deficient mice were examined, a significant decrease in FceRI- 
mediated degranulation, but not phorbol 12-myristate 13-acetate/ionomycin-induced degranulation was observed. These data suggested that CD63 may be required for generation of so far undefined FceRI proximal signalling events that feed membrane fusion processes. Reconstitution of mast cell-deficient $K i t^{w / W-v}$ mice with CD63-deficient mast cells led to significantly attenuated PCA, confirming a positive regulatory role for CD63 on mast degranulation under physiological conditions [38]. How exactly CD63 regulates FcERI signalling is not known, but it could be related to the finding that CD63 can recruit the calcium sensor synaptotagmin VII to lysosomes [39].

A different phenotype was observed in BMMCs from $\mathrm{CD} 151^{-/-}$mice [40], which exhibited normal antigen-induced degranulation, but significantly enhanced expression of the proinflammatory cytokines TNF- $\alpha$, IL-4 and IL-13. Mice deficient in CD151 exhibited an enhanced PCA reaction, supporting the concept that CD151 is a negative regulator of the FcعRI-mediated signalling events in mast cells. Additional mechanistic studies showed that CD151 negatively regulated the ERK1/2-PI3 kinase-AKT signalling pathway without affecting phospholipase $C_{\gamma} 1$ activation and calcium response [40].

Although mice deficient in CD9 expression were generated more than 17 years ago [41], studies on $\mathrm{CD}^{-/}$mast cells are very limited. Interestingly, when wild-type BMMCs were exposed to a CD9-specific mAb, $2 \mathrm{H9}$, the cells exhibited enhanced calcium mobilization and degranulation [42]. Antibody-mediated aggregation of CD9 also induced enhanced tyrosine phosphorylation of ERK, p38 MAP kinase and the transmembrane adaptor proteins LAT1 and especially LAT2. 2H9 mAb inhibited chemotaxis towards antigen. This inhibitory effect was observed after treatment with $\mathrm{F}(\mathrm{ab})_{2}$ fragment but not with Fab fragment, suggesting that chemotaxis inhibition requires CD9 aggregation but is independent of co-cross-linking of CD9 with the inhibitory Fc $\gamma$ RIIB (that is specific for the Fc portion absent in $F(a b)_{2}$ fragments). In BMMCs with siRNA-mediated CD9 knockdown, migration towards antigen was not affected [42]. The observed inhibition of antigen-mediated chemotaxis by $2 \mathrm{H} 9 \mathrm{mAb}$ or its $\mathrm{F}(\mathrm{ab})_{2}$ fragment could be related to the ability of CD9 to associate with integrin molecules $[43,44]$.

A recent study showed that CD9 and CD63 are not equally distributed on extracellular vesicles released from mast cells [45]. Vesicles constitutively released from unstimulated 
cells were CD9 positive, whereas vesicles released from antigen-activated cells were smaller, CD63 positive, and exhibited a higher buoyant density, distinct phospholipid composition, and higher amount of mast cell specific proteases. These and other findings [46] suggested that the tetraspanins CD9 and CD63 could be involved in sorting distinct vesicles with different immunological functions. The combined data provide evidence that members of the tetraspanin family have specific non-redundant functions in IgE-mediated signalling and other mast cell functions. How exactly, in molecular terms, tetraspanins interfere with FceRIand other surface receptor-triggered activation events is not completely understood. The transmembrane domains of the tetraspanins support their interactions with other tetraspanins and a broad array of other molecules in the cell membrane, and the formation of tetraspanin-enriched microdomains. Recent data suggested that in immune cells, individual tetraspanins form homoclusters of less than 10 tetraspanin molecules per cluster and that clusters possessing different tetraspanins interact with different membrane receptors [47]. Through these interactions different tetraspanins could regulate various signal transduction events in different way.

\section{The CD300 family: paired inhibitory and activating receptors}

One of the most striking groups of receptors found on mast cells is the paired receptors, which include activating receptors (ARs) and inhibitory receptors (IRs) [48]. They usually bind the same ligand, since in most cases the extracellular portion of these receptors is highly similar. A rule of thumb is that the affinity of the IRs is always higher as compared to that of the ARs and therefore inhibition is always dominant. Usually the paired receptors bind self and non-self-ligands to maintain homeostasis. The IRs possess a long tail and inhibitory ITIMs. In contrast, the ARs have short cytoplasmic regions and a positively charged residue in the transmembrane domain that is associated with an adaptor protein possessing an activating ITAM (see Box 1).

The CD300 family of receptors has eight human members and nine mouse members. This family contains IRs and ARs, and is expressed by many immune cells, and notably by mast cells [49]. Of the CD300 family, human and mouse CD300a and CD300c are the only welldefined paired receptors, sharing around $80 \%$ homology. Importantly, three members of the human (CD300a, CD300c and CD300f) and four of the mouse CD300 family, namely CD300a 
(LMIR-1), CD300If (CD300f, CLM-1, DIgR2, LMIR-3, MAIR-V), CD300lb (LMIR-5) and CD300lh (LMIR7, CLM-3), are expressed and functional on mast cells ([50], Table 1, and Figure 1C).

The best-defined ligands for human CD300a are phosphatidylserine (PS) and phosphatidylethanolamine (PE) [50]. PS and PE are expressed on apoptotic cells, on tumour cells and on the external leaflet of the plasma membrane of activated and viral infected cells, suggesting CD300a-mediated regulation under these conditions. Additional ligands for the CD300 members are ceramide, TIM1 (T-cell immunoglobulin and mucin domain 1) and sphingolipids (Figure 1C).

Munitz, A et al., were the first to demonstrate that CD300a (recently reviewed in [51]) is displayed on mast cells, eosinophils and basophils, acting to decrease their FceRI-mediated activation [52-55]. Later, it was demonstrated that DNAM1 (an activating receptor)mediated activation of mast cells is completely blocked by CD300a activation [56]. The mechanisms of the inhibition rely on the anti-CD300a induced phosphorylation of the phosphatases SHP-1 and SHIP-1 and consequently dephosphorylation of a number of signal transduction molecules including SYK, LAT and KIT $[49,50]$. Furthermore, it was shown, using chemically-generated bi-specific antibodies against CD300a and IgE and against CD300a and KIT, that CD300a inhibits mast cell activation and survival mediated by IgE-dependent mechanisms and by stem cell factor (SCF) $[57,58]$. Therefore, selective targeting of CD300a on mast cells could be an effective immune-pharmacological method of treating mast cellassociated diseases.

Using a CD300c specific mAb [59] it was shown that CD300c is highly expressed in human mast cells and that after cross-linking leads to cytokine production. Furthermore, it was demonstrated that both $\mathrm{CD} 300 \mathrm{a}$ and $\mathrm{CD} 300 \mathrm{c}$ interact with $\mathrm{PE}$ producing a dominant inhibitory interaction [59]. The same group also found that CD300f, whose ligands are sphingomyelin and ceramide, is expressed on mast cells, inhibiting their Fc\&RI activation [60].

CD300a is expressed on mouse mast cells and in a model of allergic peritonitis its neutralization with specific antibodies resulted in a significant increase in inflammatory mediators and eosinophilic infiltration [52]. On the other hand, specific targeting of CD300a 
with IgE or with CC chemokine receptor 3 (CCR3), using a bispecific antibody, significantly decreased airway inflammation and tissue remodelling and SCF induced cutaneous anaphylaxis $[57,61]$.

CD300a knockout mice were generated by the Shibuya group [62], who showed that CD300a delivers an inhibitory signal in mast cells to suppress production of lipopolysaccharide (LPS)induced inflammatory cytokines and chemokines. Surprisingly, an opposite picture was seen in an experimental allergic airway inflammation model induced by alum with ovalbumin. In CD300a-deficient mice, eosinophil infiltration, serum IgE levels, and airway hyper-reactivity were significantly decreased after challenge [63], possibly because dendritic cells in the CD300a-deficient mice produced less IL-4.

CD300lf is another negative regulator of mast cell activation following interaction with ceramide [64] and PS [65]. Under certain conditions, CD300If can amplify immune responses [66]. The intracellular tail of CD300If has both ITIM and activating motifs, like PI3K binding motifs, highlighting the complexity in the ligand binding and signalling properties mediated by the CD300 family of receptors. Indeed, CD300lf-deficient mice showed enhanced allergic responses including anaphylaxis, airway inflammation, and dermatitis [67]. Interestingly, it was recently demonstrated that mast cells play an important role in dextran sulphate sodium (DSS)-induced colitis, as in the absence of CD300If (the experiment included transplanted mice lacking CD300IF only in mast cells), colitis in mice was exacerbated [68]. Furthermore, it was reported that ceramide-CD300If interactions inhibited ATP-stimulated activation of BMMCs [68]. In contrast, upon association with FcRy it switches its function to become an activating receptor in the presence of TLR4 stimulation [69].

CD300lb, is another CD300 member expressed on mast cells and is a DAP12-coupled AR, binding TIM1, PS and LPS [70]. Stimulation with TIM1 induced CD300lb mediated activation of mast cells [71]. Cross-linking of CD300lb induced SYK-dependent activation of fetal liverderived mast cells [72]. CD300lh is highly expressed in BMMCs and found to associate with FcR $y$ and its engagement induced cytokine production [73]. 
While it is still unclear why CD300f and CD300a enhances mast cell activity under certain conditions this emphasizes the need for the generation of cell specific knockout mice of all of the above mentioned receptors and the necessity to fully understand the signalling mechanisms governing the activities of the CD300 receptors. Furthermore, it might be that under certain conditions, other members of the CD300 family are expressed, and/or pair with each other, or even bind additional ligands. Certainly, more research is required to fully understand the exact function of the CD300 family in controlling immune responses in general and allergy in particular.

\section{Broad-Ranging Ligands Reconvening to a Shared MRGX2 Receptor-Mediated Signalling}

Mast cells express several G protein-coupled receptors (GPCRs), which constitute the largest class of receptors in eukaryotes (i.e. approximately 800 identified in humans [74]). Several of the well-known mast cell secretagogues, e.g., complement, neuropeptides, endothelin, host defense peptides or venoms bind to a GPCR and induce mast cell exocytosis and release of mediators. One GPCR that has received a lot of attention lately is the Mas-related G proteincoupled receptor X2 (MRGPRX2) [75]. MRGPRX2 was first identified in neurons of the dorsal root ganglion, and later it was recognized that mast cells appear to be the only cell besides dorsal root ganglion to express this receptor. Detailed transcriptomic analysis revealed that MRGPRX2 is selectively and highly expressed in human skin mast cells [76]. Similarly, the mouse ortholog MRGPRB2, is selectively expressed in mast cells [77]. Hence, besides its expression in dorsal root ganglia, MRGPRX2 has emerged as a unique and specific mast cell receptor.

MRGPRX2 appears to be mainly expressed in the $\mathrm{MC}_{\mathrm{Tc}}$ type of mast cells [78], which is the predominant mast cell type of the skin. In the lung and gut the tryptase-only containing mast cells $\mathrm{MC}_{\mathbf{T}}$ dominate, although some $\mathrm{MC}_{\mathrm{TC}}$ can be found in lower frequency. Although heterogeneity in reactivity to basic peptides, such as compound 48/80 and substance $P$, between $\mathrm{MC}_{\mathrm{TC}}$ and $\mathrm{MC}_{\mathrm{T}}$ has been recognized for a long time [79], it was with the discovery of the MRGPRX2 that a mechanism for this heterogeneity was revealed [78]. Besides MRGPRX2, there are indications that substance $P$ might also activate mast cells through the substance P receptor Neurokinin 1-receptor [80]. Several other peptides activate mast cells through MRGPRX2 including somatostatin, angiopeptin, mast cell degranulating peptide 
(MCDP) [78] and host defense peptides such as $\beta$-defensins [81], as well as eosinophil major basic protein and peroxidase [82]. Of particular interest is that pseudoallergic responses to peptidergic-drugs are induced through activation of MRGPRX2 [83]. For example, icatibant, a bradykinin B2 receptor antagonist used for the treatment of hereditary angioedema, causes mast cell activation and release of histamine [84]. Other examples are neuromuscular blocking drugs when injected to reduce muscle movement during surgery [85]. In addition, antibiotics of the fluoroquinolone family can also induce histamine release and allergic reactions [86]. Using a mouse model where the mouse ortholog to the human MRGPRX2, the MRGPRB2, was mutated, McNeil et al. could demonstrate that these three classes of drugs activate mast cells through MRGPRB2 and that mice lacking functional MRGPRB2 exhibit significantly reduced in vivo responses to these drugs compared with wild-type mice [83].

In contrast to allergic, IgE-FcERI-dependent, cutaneous reactions that often are long-lasting, pseudoallergic reactions, i.e., MRGPRX2-dependent, appear to be more transient. A plausible explanation for this apparent difference between these two activation pathways was described, recently [87]. Substance P, which activates MRGPRX2, induced a rapid, almost immediate, secretion of small and relatively spherical granules, presumably representing individual mast cell granules (Fig. 1D). In contrast, FceRI-mediated activation caused a delayed secretion of larger and more heterogeneously shaped granules, most likely due to granule fusion. Inhibition of IKK- $\beta$ during the IgE-dependent activation, inhibited the SNAP23/Syntaxin-4 fusion process, and switched the pattern into one that resembled the substance P-induced mast cell degranulation. In vivo studies demonstrated that MRGPRX2mediated degranulation induced a more rapid and transient vascular reaction compared to the more intense and prolonged response after Fc\&RI-activation [87].

The identification of MRGPRX2 as a mast cell restricted receptor that induces degranulation and release of mediators in a distinct fashion with spherical granules that appear to be unstable, in contrast to the fused granules that are released after IgE-dependent activation (Figure 1D), has provided important information about the function of this receptor in health and disease. Besides pseudo-allergic reactions induced by certain drugs, this receptor is most likely involved in pathological conditions such as neurogenic inflammations and chronic urticaria $[78,82]$. Skin mast cells in patients with chronic urticaria express increased levels of 
MRGPRX2 [82]. Together with the findings that substance $P$ and eosinophil-released products activate skin mast cells via MRGPRX2 this suggests that this pathway contributes to the pathogenesis [82]. Thus, MRGPRX2 might be a new target molecule for the treatment of wheal reactions in patients with severe chronic urticaria and for circumvention of pseudoallergic reactions upon treatment with certain drugs. Whether the activity of the GPCRs, including MRGPRX2, expressed on mast cells are regulated by the tetraspanins and the IRs and ARs discussed above remains to be elucidated.

\section{Concluding Remarks and Future Perspectives}

In this review, we have highlighted negative and positive signals that control mast cell activation, focussing on modalities which control FcERI signalling and on IgE-independent pathways such as the one driven by MRGX2 receptor engagement. We synthesize a complex FceRI signalling network where CD300 family members, using proper self-initiated, allergenindependent signalling, act as co-receptors to potentiate or dampen allergen-mediated mast cell activation.

It is becoming evident that a defined spatial organization involving the compartmentalization of receptors in specialized mast cell membrane domains and their linkage to the underlying cytoskeleton and signalling molecules is essential for proper activation. In mast cells, tetraspanins such as CD9, CD63, CD81 and CD151 not only serve as such guarantors of cell membrane structural integrity, but also exhibit significant crosstalk with FceRI signalling events.

What remains still largely unclear are the internal signalling routes that decide on signal diversification by channelling the information from activated receptors into distinct biological functions such as preferential degranulation versus mediator secretion. Likewise, the direct or indirect "ranking" of signals that control synchronization, strength and velocity of mast cell activation or desensitization are still ill-defined, and the hierarchy and choreography of these signals in defined mast cell populations and signalling milieus remains essentially unknown and may be difficult to predict. Likely, to better grasp this, a systems biology approach to mast cell activation will be indispensable. 
Indisputably, heterogeneous, highly plastic and adaptable mast cells have revealed themselves as functionally very flexible in distinct tissue environments. The major open question at hand here is which key mechanisms that control mast cell activation are shared and thus universal, and which ones are strictly, context-, location-, and cell populationdependent? Another central open question is which of these activation controls can be considered as physiological, and which ones are pathological (see outstanding questions).

Evidently, with an armamentarium of modern methods, including CRISPR/Cas techniques, next-generation sequencing, superresolution microscopy, single-cell and single-molecule analyses, computational modeling, and quantitative proteomic analysis, understanding of complex signalling pathways will be accelerated over the next few years and many puzzling findings will be clarified. For example, for many years there was an apparent discrepancy between the activities of PAG adaptor proteins in T cells and those in mast cells. In T cells PAG is constitutively phosphorylated but becomes dephosphorylated following $T$ cell receptor (TCR) activation. However, in mast cells PAG phosphorylation occurs following FceRI activation. This discrepancy was important because PAG phosphorylation is thought to anchor C-terminal Src kinase, an inhibitor of Src kinases, which should therefore prevent rather than promote, FceRI signalling in activated mast cells [88]. This matter has been recently resolved using quantitative phosphoproteomic analysis. In this study it was shown that TCR-mediated activation of resting T cells leads to enhanced PAG phosphorylation [89], as seen in mast cells indicating that PAG could have both positive and negative roles in immunoreceptor signalling [90]. Another example of the facility of new technologies in mast cell biology is the recent identification, using next generation sequncing, of distinct extracellular RNA signatures released by mast cells in culture [91]. New methodological approaches will undoubtedly deepen our understanding of the molecular pathobiology of allergies and other inflammatory diseases and will facilitate the development of new drugs for mast-cell mediated diseases.

\section{Acknowledgments}

This work was supported by Czech Science Foundation, project P302/12/G101 (P.D.); the Swedish Research Council and Karolinska Intitutet (G.N.); the Israel Science Foundation, the 
Binational Israel-USA Foundation and the Rosetrees Foundation (FLS); the Institut National de la Recherche Medicale (INSERM), the Centre National de la Recherche Scientifique (CNRS) and the "Investissements d'Avenir programme ANR-11-IDEX-0005-02, Sorbonne Paris Cite, Laboratoire d'excellence INFLAMEX, Paris France (U.B.). We thank Martin Barron and Rajia Bahri for critical reading of the manuscript. 


\section{Figure Legend}

Figure 1: Regulatory Mechanisms Controlling Mast Cell Activation. A. Concentration [16] and binding affinity of allergens [19] modulate FcERI-induced mast cell activities. B. The tetraspanins CD9, CD63, CD81, and CD151 display positive or negative regulatory roles on FceRI-mediated mast cell signalling. C. The CD300 family includes activating receptors and inhibitory receptors. PS: phosphatidylserine, PE: phosphatidylethanolamine, SM: sphingomyelin, TIM-1: T-cell immunoglobulin and mucin domain 1. D. The MRGPRX2 receptor induces degranulation and release of mediators in a distinct fashion with spherical granules differently from the IgE-dependent activation. 


\section{$\underline{\text { References }}$}

1 St John, A.L. and Abraham, S.N. (2013) Innate immunity and its regulation by mast cells. J Immunol $190,4458-4463$

2 Mukai, K., et al. (2016) IgE and mast cells in host defense against parasites and venoms. Semin Immunopathol 38, 581-603

3 Bulfone-Paus, S. and Bahri, R. (2015) Mast Cells as Regulators of T Cell Responses. Front Immunol 6, 394

4 Rivera, J. and Gilfillan, A.M. (2006) Molecular regulation of mast cell activation. J Allergy Clin Immunol 117, 1214-1225; quiz 1226

5 Galli, S.J., et al. (2008) Immunomodulatory mast cells: negative, as well as positive, regulators of immunity. Nat Rev Immunol 8, 478-486

6 Sibilano, R., et al. (2014) Mast cell activation: a complex interplay of positive and negative signaling pathways. Eur J Immunol 44, 2558-2566

7 Christensen, L.H., et al. (2008) Several distinct properties of the IgE repertoire determine effector cell degranulation in response to allergen challenge. J Allergy Clin Immunol 122, 298-304

8 Gadermaier, E., et al. (2014) The human IgE repertoire. Int Arch Allergy Immunol 163, 77-91

9 Altrichter, S., et al. (2011) IgE mediated autoallergy against thyroid peroxidase--a novel pathomechanism of chronic spontaneous urticaria? PLoS One 6, e14794

10 Dema, B., et al. (2014) Immunoglobulin E plays an immunoregulatory role in lupus. J Exp Med 211, 2159-2168

11 Sanjuan, M.A., et al. (2016) Role of IgE in autoimmunity. J Allergy Clin Immunol 137, 1651-1661

12 Segal, D.M., et al. (1977) Dimeric immunoglobulin E serves as a unit signal for mast cell

degranulation. Proc Natl Acad Sci U S A 74, 2993-2997

13 Asai, K., et al. (2001) Regulation of mast cell survival by IgE. Immunity 14, 791-800

14 Kalesnikoff, J., et al. (2001) Monomeric IgE stimulates signaling pathways in mast cells that lead to cytokine production and cell survival. Immunity 14, 801-811

15 Kitaura, J., et al. (2003) Evidence that IgE molecules mediate a spectrum of effects on mast cell survival and activation via aggregation of the FceRI. Proc Natl Acad Sci U S A 100, 12911-12916

16 Gonzalez-Espinosa, C., et al. (2003) Preferential signaling and induction of allergy-promoting lymphokines upon weak stimulation of the high affinity IgE receptor on mast cells. J Exp Med 197, 1453-1465

17 Marchand, F., et al. (2003) Human serum IgE-mediated mast cell degranulation shows poor correlation to allergen-specific IgE content. Allergy 58, 1037-1043

18 Torigoe, C., et al. (1998) An unusual mechanism for ligand antagonism. Science 281, 568-572

19 Suzuki, R., et al. (2014) Molecular editing of cellular responses by the high-affinity receptor for IgE. Science 343, 1021-1025

20 Gieras, A., et al. (2016) IgE epitope proximity determines immune complex shape and effector cell activation capacity. J Allergy Clin Immunol 137, 1557-1565

21 Gieras, A., et al. (2007) Molecular determinants of allergen-induced effector cell degranulation. $J$ Allergy Clin Immunol 119, 384-390

22 Shalit, M. and Levi-Schaffer, F. (1995) Challenge of mast cells with increasing amounts of antigen induces desensitization. Clin Exp Allergy 25, 896-902

23 Liu, A., et al. (2011) Desensitization regimens for drug allergy: state of the art in the $21^{\text {st }}$ century.

Clin Exp Allergy 41, 1679-1689

24 Sancho-Serra Mdel, C., et al. (2011) Rapid IgE desensitization is antigen specific and impairs early and late mast cell responses targeting FcERI internalization. Eur J Immunol 41, 1004-1013

25 Oka, T., et al. (2002) FceRI cross-linking-induced actin assembly mediates calcium signalling in RBL$2 \mathrm{H} 3$ mast cells. Br J Pharmacol 136, 837-846

26 Ang, W.X., et al. (2016) Mast cell desensitization inhibits calcium flux and aberrantly remodels actin. J Clin Invest 
27 Khodoun, M.V., et al. (2013) Rapid polyclonal desensitization with antibodies to IgE and FceRIa. J Allergy Clin Immunol 131, 1555-1564

28 Blank, U., et al. (2009) Inhibitory ITAMs as novel regulators of immunity. Immunol Rev 232, 59-71 29 Aloulou, M., et al. (2012) IgG1 and IVIg induce inhibitory ITAM signaling through FcyRIII controlling inflammatory responses. Blood 119, 3084-3096

30 Kanamaru, Y., et al. (2008) Inhibitory ITAM signaling by FcaRI-FcR $\gamma$ chain controls multiple activating responses and prevents renal inflammation. J Immunol 180, 2669-2678

31 Pfirsch-Maisonnas, S., et al. (2011) Inhibitory ITAM signaling traps activating receptors with the phosphatase SHP-1 to form polarized "inhibisome" clusters. Sci Signal 4, ra24

32 Andrews, N.L., et al. (2008) Actin restricts FceRI diffusion and facilitates antigen-induced receptor immobilization. Nat Cell Biol 10, 955-963

33 Hemler, M.E. (2005) Tetraspanin functions and associated microdomains. Nat Rev Mol Cell Biol 6, 801-811

34 Yanez-Mo, M., et al. (2009) Tetraspanin-enriched microdomains: a functional unit in cell plasma membranes. Trends Cell Biol 19, 434-446

35 Halova, I. and Draber, P. (2016) Tetraspanins and Transmembrane Adaptor Proteins As Plasma Membrane Organizers-Mast Cell Case. Front Cell Dev Biol 4, 43

36 Fleming, T.J., et al. (1997) Negative regulation of FcERI-mediated degranulation by CD81. J Exp Med 186, 1307-1314

$37 \mathrm{Kraft}$, S., et al. (2005) Anti-CD63 antibodies suppress IgE-dependent allergic reactions in vitro and in vivo. J Exp Med 201, 385-396

$38 \mathrm{Kraft}$, S., et al. (2013) The tetraspanin CD63 is required for efficient IgE-mediated mast cell degranulation and anaphylaxis. J Immunol 191, 2871-2878

39 Flannery, A.R., et al. (2010) Palmitoylation-dependent association with CD63 targets the $\mathrm{Ca}^{2+}$ sensor synaptotagmin VII to lysosomes. J Cell Biol 191, 599-613

40 Abdala-Valencia, H., et al. (2015) Tetraspanin CD151 Is a Negative Regulator of Fc\&RI-Mediated Mast Cell Activation. J Immunol 195, 1377-1387

41 Le Naour, F., et al. (2000) Severely reduced female fertility in CD9-deficient mice. Science 287, 319-321

42 Halova, l., et al. (2013) Cross-talk between tetraspanin CD9 and transmembrane adaptor protein non-T cell activation linker (NTAL) in mast cell activation and chemotaxis. J Biol Chem 288, 9801-9814 43 Bassani, S. and Cingolani, L.A. (2012) Tetraspanins: Interactions and interplay with integrins. Int J Biochem Cell Biol 44, 703-708

44 Reyes, R., et al. (2015) Different states of integrin LFA-1 aggregation are controlled through its association with tetraspanin CD9. Biochim Biophys Acta 1853, 2464-2480

45 Groot Kormelink, T., et al. (2016) Mast Cell Degranulation Is Accompanied by the Release of a Selective Subset of Extracellular Vesicles That Contain Mast Cell-Specific Proteases. J Immunol 46 Kowal, J., et al. (2016) Proteomic comparison defines novel markers to characterize heterogeneous populations of extracellular vesicle subtypes. Proc Natl Acad Sci U S A 113, E968-977 47 Zuidscherwoude, M., et al. (2015) The tetraspanin web revisited by super-resolution microscopy. Sci Rep 5, 12201

48 Martinet, L. and Smyth, M.J. (2015) Balancing natural killer cell activation through paired receptors. Nat Rev Immunol 15, 243-254

49 Karra, L., et al. (2009) Are we ready to downregulate mast cells? Curr Opin Immunol 21, 708-714 50 Borrego, F. (2013) The CD300 molecules: an emerging family of regulators of the immune system. Blood 121, 1951-1960

51 Zenarruzabeitia, O., et al. (2015) The Biology and Disease Relevance of CD300a, an Inhibitory Receptor for Phosphatidylserine and Phosphatidylethanolamine. J Immunol 194, 5053-5060 52 Bachelet, l., et al. (2005) The inhibitory receptor IRp60 (CD300a) is expressed and functional on human mast cells. J Immunol 175, 7989-7995

53 Munitz, A., et al. (2006) The inhibitory receptor IRp60 (CD300a) suppresses the effects of IL-5, GM-CSF, and eotaxin on human peripheral blood eosinophils. Blood 107, 1996-2003 
54 Gibbs, B.F., et al. (2012) Expressions and inhibitory functions of CD300a receptors on purified human basophils. Exp Dermatol 21, 884-886

55 Sabato, V., et al. (2012) CD300a is expressed on human basophils and seems to inhibit IgE/Fc\&RIdependent anaphylactic degranulation. Cytometry B Clin Cytom 82, 132-138

56 Bachelet, l., et al. (2006) Mast cell costimulation by CD226/CD112 (DNAM-1/Nectin-2): a novel interface in the allergic process. J Biol Chem 281, 27190-27196

57 Bachelet, l., et al. (2006) Abrogation of allergic reactions by a bispecific antibody fragment linking IgE to CD300a. J Allergy Clin Immunol 117, 1314-1320

58 Bachelet, l., et al. (2008) Suppression of normal and malignant kit signaling by a bispecific antibody linking kit with CD300a. J Immunol 180, 6064-6069

59 Takahashi, M., et al. (2013) Human CD300C delivers an Fc receptor- $\gamma$-dependent activating signal in mast cells and monocytes and differs from CD300A in ligand recognition. J Biol Chem 288, 7662-

7675

60 Izawa, K., et al. (2014) Sphingomyelin and ceramide are physiological ligands for human

LMIR3/CD300f, inhibiting Fc\&RI-mediated mast cell activation. J Allergy Clin Immunol 133, 270-273 e271-277

61 Munitz, A., et al. (2006) Reversal of airway inflammation and remodeling in asthma by a bispecific antibody fragment linking CCR3 to CD300a. J Allergy Clin Immunol 118, 1082-1089

62 Nakahashi-Oda, C., et al. (2012) Apoptotic cells suppress mast cell inflammatory responses via the CD300a immunoreceptor. J Exp Med 209, 1493-1503

63 Miki, H., et al. (2015) Involvement of CD300a Phosphatidylserine Immunoreceptor in Aluminum

Salt Adjuvant-Induced Th2 Responses. J Immunol 194, 5069-5076

64 Izawa, K., et al. (2007) Functional analysis of activating receptor LMIR4 as a counterpart of inhibitory receptor LMIR3. J Biol Chem 282, 17997-18008

65 Voss, O.H., et al. (2015) Emerging role of CD300 receptors in regulating myeloid cell efferocytosis. $\mathrm{Mol}$ Cell Oncol 2, e964625

66 Moshkovits, I., et al. (2015) CD300f associates with IL-4 receptor alpha and amplifies IL-4-induced immune cell responses. Proc Natl Acad Sci U S A 112, 8708-8713

67 Izawa, K., et al. (2012) The receptor LMIR3 negatively regulates mast cell activation and allergic responses by binding to extracellular ceramide. Immunity $37,827-839$

68 Matsukawa, T., et al. (2016) Ceramide-CD300f binding suppresses experimental colitis by

inhibiting ATP-mediated mast cell activation. Gut 65, 777-787

69 Izawa, K., et al. (2009) An activating and inhibitory signal from an inhibitory receptor LMIR3/CLM-

1: LMIR3 augments lipopolysaccharide response through association with FcR $\gamma$ in mast cells. J

Immunol 183, 925-936

70 Voss, O.H., et al. (2016) Lipopolysaccharide-Induced CD300b Receptor Binding to Toll-like

Receptor 4 Alters Signaling to Drive Cytokine Responses that Enhance Septic Shock. Immunity 44, 1365-1378

71 Yamanishi, Y., et al. (2010) TIM1 is an endogenous ligand for LMIR5/CD300b: LMIR5 deficiency ameliorates mouse kidney ischemia/reperfusion injury. J Exp Med 207, 1501-1511

72 Yamanishi, Y., et al. (2008) Analysis of mouse LMIR5/CLM-7 as an activating receptor: differential regulation of LMIR5/CLM-7 in mouse versus human cells. Blood 111, 688-698

73 Enomoto, Y., et al. (2010) Characterization of leukocyte mono-immunoglobulin-like receptor 7

(LMIR7)/CLM-3 as an activating receptor: its similarities to and differences from LMIR4/CLM-5. J Biol Chem 285, 35274-35283

74 Vassilatis, D.K., et al. (2003) The G protein-coupled receptor repertoires of human and mouse.

Proc Natl Acad Sci U S A 100, 4903-4908

75 Subramanian, H., et al. (2016) Roles of Mas-related G protein-coupled receptor X2 on mast cellmediated host defense, pseudoallergic drug reactions, and chronic inflammatory diseases. J Allergy Clin Immunol 138, 700-710

76 Motakis, E., et al. (2014) Redefinition of the human mast cell transcriptome by deep-CAGE sequencing. Blood 123, e58-67 
77 Dwyer, D.F., et al. (2016) Expression profiling of constitutive mast cells reveals a unique identity within the immune system. Nat Immunol 17, 878-887

78 Tatemoto, K., et al. (2006) Immunoglobulin E-independent activation of mast cell is mediated by Mrg receptors. Biochem Biophys Res Commun 349, 1322-1328

79 Lowman, M.A., et al. (1988) Human mast cell heterogeneity: histamine release from mast cells dispersed from skin, lung, adenoids, tonsils, and colon in response to IgE-dependent and nonimmunologic stimuli. J Allergy Clin Immunol 81, 590-597

80 Cao, T., et al. (1999) Use of NK(1) knockout mice to analyze substance P-induced edema formation. Am J Physiol 277, R476-481

81 Subramanian, H., et al. (2013) $\beta$-Defensins activate human mast cells via Mas-related gene X2. J Immunol 191, 345-352

82 Fujisawa, D., et al. (2014) Expression of Mas-related gene X2 on mast cells is upregulated in the skin of patients with severe chronic urticaria. J Allergy Clin Immunol 134, 622-633 e629

83 McNeil, B.D., et al. (2015) Identification of a mast-cell-specific receptor crucial for pseudo-allergic drug reactions. Nature 519, 237-241

84 Maurer, M. and Church, M.K. (2012) Inflammatory skin responses induced by icatibant injection are mast cell mediated and attenuated by $\mathrm{H}(1)$-antihistamines. Exp Dermatol 21, 154-155

85 Koppert, W., et al. (2001) Different patterns of mast cell activation by muscle relaxants in human skin. Anesthesiology 95, 659-667

86 Mori, K., et al. (2000) Characterization of histamine release induced by fluoroquinolone antibacterial agents in-vivo and in-vitro. J Pharm Pharmacol 52, 577-584

87 Gaudenzio, N., et al. (2016) Different activation signals induce distinct mast cell degranulation strategies. J Clin Invest 126, 3981-3998

88 Draber, P., et al. (2011) Transmembrane adaptor proteins in the high-affinity IgE receptor signaling. Front Immunol 2, 95

89 Reginald, K., et al. (2015) Revisiting the Timing of Action of the PAG Adaptor Using Quantitative Proteomics Analysis of Primary T Cells. J Immunol 195, 5472-5481

90 Draberova, L., et al. (2014) Transmembrane adaptor protein PAG/CBP is involved in both positive and negative regulation of mast cell signaling. Mol Cell Biol 34, 4285-4300

91 Lasser, C., et al. (2016) Two distinct extracellular RNA signatures released by a single cell type identified by microarray and next-generation sequencing. RNA Biol, 1-15

\section{Glossary}

FcERI: Type I high affinity IgE receptor (FcepsilonRI) expressed on mast cells and basophils and also on several other cells (eosinophils, macrophages, platelets) in humans. Responsible for type I hypersensitivity effector responses and allergies.

$\mathrm{MC}_{\mathrm{T}}$ and $\mathrm{MC}_{\mathrm{TC}}$ : Mast cells containing the protease tryptase $\left(\mathrm{MC}_{\mathrm{T}}\right)$ predominantly found at mucosal sites, e.g., in the lung and gastrointerstinal tract, and mast cells containing the proteases tryptase, chymase and carboxypetidase $A\left(M C_{T C}\right)$ which is the predominant mast cell type of the skin.

MRGPRX2: Mas-related G-protein coupled receptor X2. When the MRG-family was discovered it was found that the genes encoding this family of G-protein coupled receptors 
were related to the MAS1 oncogene and therefore the receptors are called MAS-related Gprotein coupled receptors.

ITAM: Immunoreceptor Tyrosine-based Activating Motif. Motif usually found in adaptor proteins that are associated, via charged residues in their transmembrane domain, to a charged residue present in the transmembrane domain of an activating receptor (AR). Its phosphorylation after engagement of the AR leads to recruitment of kinases, which deliver the activating signal.

ITAMi: inhibitory ITAM motif denotes the concept that the ITAM motif - under certain conditions (low valency aggregation) - can also mount an inhibitory response, which is effective towards heterologous receptors and involves recruitment of SHP-1 phosphatase.

ITIM: Immunoreceptor Tyrosine-based Inhibitory Motif. Motif found in inhibitory receptors (IRs) that upon phosphorylation promotes recruitment of phosphatases to deliver an inhibitory signal upon engagement of the receptors by their ligands.

Paired receptors: Receptors that share high homology in their extracellular domains, usually binding the same ligand. The paired receptors include at least one pair of IRs and ARs. The IR binding affinity is always higher as compared to the AR and therefore when the paired receptors are simultaneously engaged, inhibition is always dominant.

PCA: passive cutaneous anaphylaxis is a method for analysis of mast cell function in vivo. In a typical PCA, IgE is injected intradermally where it binds to and locally sensitizes mast cells. Next day antigen specific for the IgE and Evans blue are injected intravenously. Binding of the antigen to IgE-sensitized mast cells leads to mast cell degranulation, followed by increased permeability of vessels and Evans blue extravasation. 


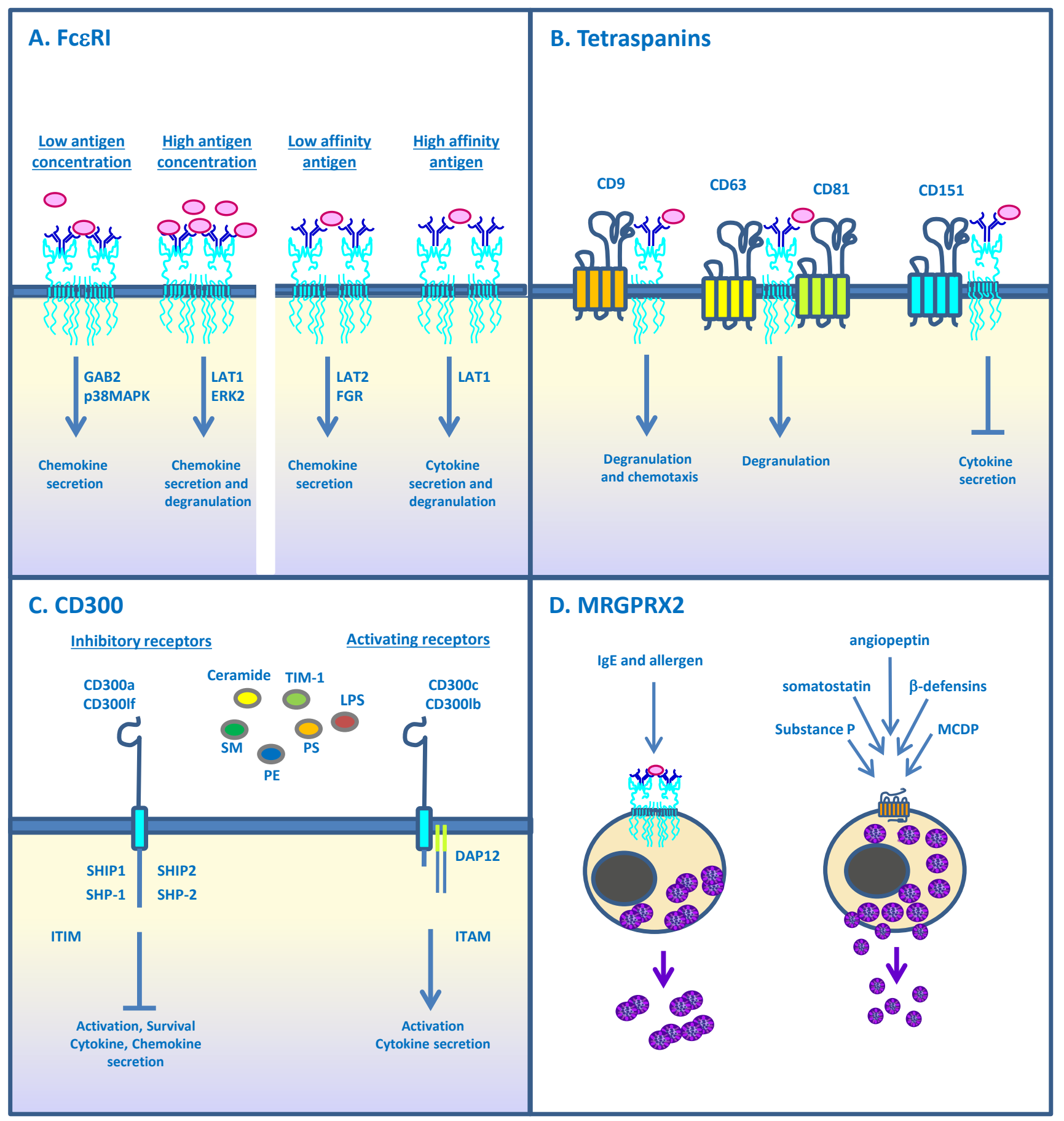




\begin{tabular}{|c|c|c|c|}
\hline Name & Species & AR or IR & Ligands \\
\hline CD300a & Human & IR & PS, PE \\
\hline CD300c & Human & AR & PS, PE \\
\hline CD300f & Human & IR & Sphingomyelin, Ceramide \\
\hline CD300a (LMIR-1) & Mouse & $\begin{array}{l}\text { IR, under certain } \\
\text { conditions can be AR }\end{array}$ & Possibly PS and PE \\
\hline $\begin{array}{l}\text { CD300If (CD300f, CLM-1, } \\
\text { DIgR2, LMIR-3, MAIR-V) }\end{array}$ & Mouse & $\begin{array}{l}\text { IR, under certain } \\
\text { conditions can be } A R\end{array}$ & Ceramide and PS \\
\hline CD300lb (LMIR-5) & Mouse & AR & TIM1, PS and LPS \\
\hline CD300lh (LMIR7, CLM-3) & Mouse & AR & Unknown \\
\hline
\end{tabular}

Table 1. CD300 members expressed by human and mouse mast cells, their activity and ligands 


\section{Trends Box}

- Although IgE effector responses mediated by Fc\&RI on mast cells have been well studied, recent evidence supports the concept that the outcome of receptor triggering is complex and fine-tuned by a number of parameters and contributing co-receptors leading to an intricate network of positive and inhibitory signals.

- The receptor MRGPRX2 is, besides dorsal root ganglia, uniquely expressed on mast cells, particularly in the skin, and reacts to basic peptides, such as neuropeptides, host defense peptides, and certain drugs by inducing rapid degranulation.

- Tetraspanins have positive or negative regulatory roles on mast cell signaling pathways depending on the pathway and tetraspanin involved.

- The CD300 family of receptors has emerged as an important family containing both activating and inhibitory receptors. CD300a is the best characterized member. 


\section{Outstanding Questions Box}

- Is ITAMi signaling involved in the process of FceRI-mediated desensitization? Is this comparable to the signalling events demonstrated for FcaRI, FcyRIII and FcyRIIA?

- What is the physiological and pathophysiological role of MRGPRX2 in mast cells? How does MRGPRX2-mediated mast cell activation, in response to specific endogenous and exogenous stimuli, impact vascular and inflammatory responses?

- How, in molecular terms, do tetraspanins interfere with FceRI and other surface receptor signalling?

- What is the full ligand spectrum of the CD300 receptor family?

- Are CD300 receptor family members differently expressed in allergy-prone individuals versus controls, thus contributing to allergic reactions?

- Are signalling cascades initiated upon CD300 receptor family engagement diverse in different immune cells? 